\title{
Specificity versus selectivity: twin aims of aptasensors in bioanalysis
}

\author{
Rituraj Dubey ${ }^{1} \&$ Ravi Bhushan*,2 \\ ${ }^{1}$ Department of Chemistry, Badri Narayan Mukteshwar College, Barhiya, Munger University, Munger, Bihar-811302, India \\ ${ }^{2}$ Department of Chemistry, Indian Institute of Technology Jammu, Jammu-181221, J\&K, India \\ *Author for correspondence: rbushfcy54@gmail.com; rbushfcy@iitr.ac.in
}

First draft submitted: 16 July 2018; Accepted for publication: 20 August 2018; Published online: 18 September 2018

Keywords: aptamer biosensors • aptasensors • bioanalysis • convergence • selective aptasensors • specific and selective receptors

In the current era of bioanalysis, the most challenging task is to overcome the tussle between specific and selective receptors as these two provide different but complementary strategies. Now the question arises, can bioanalysis be aimed without convergence of specificity and selectivity? Is it possible to converge both the strategies? Let's discuss the merits and challenges of bioanalysis in terms of specificity versus selectivity in order to find the solutions.

Specificity in its ideal form denotes the bioanalysis which is completely specific to only one analyte without recognizing any other analyte. Bioconjugation pairs (such as, streptavidin/biotin, antibodies enzymatic lock-and-key and aptamers) follow this strategy [1,2]. However, challenges have been faced due to nonavailability of target-specific tools and presence of high level of similarity between analytes. Several instances including use of lectin arrays have overcome this up to some extent [3]. On the other hand, in the selective strategy, the scientists have used an array-based approach where multiple outputs from a single input could be achieved after creating a fingerprint for that sample by developing differential interaction of each target element with the analytes of interest [4].

\section{Specificity}

In more recent years, highly specific aptamer biosensors (aptasensors), which are based primarily on antibodies or enzymatic recognition, have dominated the world of bioanalysis. We cannot forget that antibodies are prone to denaturation and cannot recognize every analyte in spite of their versatility. Aptamers are basically short chains of nucleic acids or peptides and theses can be engineered to have specific binding to a target molecule and, hence, provide an alternative strategy in terms of aptasensors for specificity strategy in bioanalysis. In principle, each single aptasensor constructed with aptamer technology has a single target, and will bind to no other, even in a complex bioanalysis medium, such as, blood serum, cell lysate or an environmental sample [5-7]. Besides, aptasensors have been utilized to detect small molecules such as sugars [8], as well as big molecules such as proteins of the HIV virus [9]. Interestingly, in all the aforementioned instances, single-target specific aptasensing approaches have been used. However, array-based strategy can be used to distinguish multiple components in the sample in an example of diagnosis of disease by monitoring the levels of more than 20 biomarkers as aptasensors [10]. The biggest challenge in this case is that, in most of the cases, we don't have an idea of what biomarkers should even be targeted, as well as, many diseases do not have associated specific recognition elements.

\section{Selectivity}

Selective aptasensors emerged due to the unavailability of specific aptasensors in the intermediate region between aforementioned single-target specific and array-based multi-component specific strategies. Lectins-sugar binding proteins are used to mark glycoproteins, or glycolipids-carbohydrate-containing biomolecules, selectively, as individual lectins are not specific to individual glycosylated biomolecules in spite of having specific marking capabilities for carbohydrates (in general). In this way, a single array-based lectin approach can be used to mark the presence of carbohydrates [3]. Thus, an output similar to both specific sensor arrays and engineered selective sensor platforms can be achieved by utilizing multidimensional data analysis via taking advantage of their cross-reactivity to give discriminatory information on the analytes present. 
Besides, selectivity also comes into play if we have to design unique aptasensors for olfaction, in terms of 'chemical nose/tongue'. In this strategy we can design arrays by using various synthetic materials in order to target specific classes of analytes and in this way can easily introduce selectivity within the targets without using an antibody or aptamer. The most unique feature of these types of designed aptasensors is their flexibility as these are synthetically created leading to a wide variety of array types. These aptasensors can also target a wide variety of biomolecules, small molecules or inorganic ions, all in the same test. Thus, due to deliverance of multidimensional information from a single experimental run, these designed array based aptasensors can detect a large number of analytes without using a large number of sensor elements [11]. Furthermore, these may lead to a generation of unique patterns that can be tied back to the composition of the sample as in the case of olfaction with the help of properly engineered cross-reactivity. Selectivity focused aptasensors give 'fingerprints' that do not readily measure multiple individual components within the whole sample and this is completely different than specific aptasensors data.

\section{Convergence of specificity \& selectivity}

Since specific aptasensors give direct information on biomarkers or other analytes that is often important in categorizing complex bioanalysis samples and selective array-based aptasensors perform well when it comes to sensing the whole sample, not just its individual components, hence one must consider the particular advantages of these strategies while choosing the appropriate one. However, convergence of both the strategies of aptasensors has the potential to provide synergy which will result into much more effective aptasensor platforms like introduction of specificity lead to limited selectivity of array-based aptasensor within a group of particularly important analytes and hence we have improved stability of the array and minimized background score. There is one more area available where both the strategies are found to be complementary if we could be able to explore the underlying aptasensing mechanisms in a hypothesis-free manner. For instance, a diseased patient's sample will give a particular aptasensor response, certainly different from a healthy patient, but the study of responsible components for the difference may lead to innovations that lie behind the origin of the disease itself, as well as the mode of operation of the array-based aptasensor.

\section{Conclusion}

In spite of the fact that most of the researchers rely on specific aptasensors for individual target analytes (e.g., biomarkers) due to their excellent success in the bioanalysis domain, we cannot ignore the growth and success of selective array-based aptasensors. We strongly believe that aptasensor design would benefit from combining the best of both the approaches in order to overcome the sensing challenges. Eventually, we encourage array-based aptasensor bioanalysts to consider applying specific aptasensors to their samples in tandem and vice versa.

Financial \& competing interests disclosure

The authors have no relevant affiliations or financial involvement with any organization or entity with a financial interest in or financial conflict with the subject matter or materials discussed in the manuscript. This includes employment, consultancies, honoraria, stock ownership or options, expert testimony, grants or patents received or pending, or royalties.

No writing assistance was utilized in the production of this manuscript.

\section{References}

1. Peng HP, Lee KH, Jian JW, Yang AS. Origins of specificity and affinity in antibody-protein interactions. Proc. Natl Acad. Sci. USA 111(26), E2656-E2665 (2014).

2. Wilchek M, Bayer EA, Livnah O. Essentials of biorecognition: the (strept) avidin-biotin system as a model for protein-protein and protein-ligand interaction. Immunol. Lett. 103(1), 27-32 (2006).

3. Hirabayashi J, Yamada M, Kuno A, Tateno H. Lectin microarrays: concept, principle and applications. Chem. Soc. Rev. 42(10), 4443-4458 (2013).

4. Anzenbacher P, Palacios MA. Array-based sensors. In: Anslyn EV, Wang B (Eds). Chemosensors: Principles, Strategies, and Applications. John Wiley \& Sons, NJ, USA, 345-368 (2011).

5. Gown AM. Diagnostic immunohistochemistry: what can go wrong and how to prevent it. Arch. Pathol. Lab. Med. 140(9), 893-898 (2016).

6. Wide L, Gemzell CA. An immunological pregnancy test. Eur. J. Endocrinol. 35, 261-267 (1960).

7. Heller A, Feldman B. Electrochemical glucose sensors and their applications in diabetes management. Chem. Rev. 108(7), 2482-2505 (2008). 
8. Yang KA, Barbu M, Halim M et al. Recognition and sensing of low-epitope targets via ternary complexes with oligonucleotides and synthetic receptors. Nat. Chem. 6(11), 1003-1008 (2014).

9. Yamamoto R, Kumar PKR. Molecular beacon aptamer fluoresces in the presence of tat protein of HIV-1. Genes Cells 5(5), 389-396 (2000).

10. Lin D, Cohen FG, Hollander Z et al. Plasma protein biosignatures for detection of cardiac allograft vasculopathy. J. Heart Lung Transplant. 32(7), 723-733 (2013).

11. Liu Y, Minami T, Nishiyabu R, Wang Z, Anzenbacher P. Sensing of carboxylate drugs in urine by a supramolecular sensor array. J. Am. Chem. Soc. 135(20), 7705-7712 (2013). 
